\title{
AN ACTIVE REGION JET AND ASSOCIATED TYPE III RADIO BURST
}

\author{
S. M. Mulay*, D. Tripathi ${ }^{\dagger}$ G. Del Zanna*, and H. Mason*
}

\begin{abstract}
Extended Abstract
We present multiwavelength observations of an active region (AR) jet and its associated activity such as a nonthermal type III radio burst and changes in the photospheric magnetic field structure. The temporal and spatial correlation between the EUV jet and the type III radio burst were investigated using space-based instruments. The temperature structure of the jets and further details are reported in Mulay et al. [2016].
\end{abstract}

The Atmospheric Imaging Assembly (AIA) instrument onboard the Solar Dynamic Observatory (SDO) [Lemen et al., 2012] observed a jet originating from the western edge of the NOAA AR 11106 (S20 W09). Figure 1 (top panel) shows the AIA $171 \AA$ images of the jet activity. The jet started at 00:17 UT and lasted until 00:31 UT. A small brightening was observed at the footpoint region before the jet at 00:16 UT (top left panel). The jet appeared as a thin, collimated flow of plasma in all AIA channels, and the spire structure of the jet is shown by black arrows in the AIA $171 \AA$ images.

Photospheric magnetic activity during the jet was recorded by the Helioseismic Magnetic Imager (HMI) [Scherrer et al., 2012] onboard SDO. Figure 1 (bottom panel) shows line-of-sight HMI magnetograms before (left panel), during (middle panel), and after (right panel) the jet activity. The white arrows show the magnetic field at the footpoint region of the jet. The emergence of positive polarity was observed at the footpoint region. Also, there is a small negative polarity cancellation observed in the later phase of the jet.

The high cadence observation (12 s) of the AIA instrument enabled us to examine the jet activity in greater detail. We calculated the velocity of the jet by using a time-distance analysis technique. We drew an artificial slit along the jet-spire structure and obtained a time-distance plot during the jet activity. Figure 2 (left panel) shows the AIA $171 \AA$ image at 00:25 UT, and the white line indicates the slit position. The white dashed line in the time-distance plot (right panel of Figure 2) was used to calculate the velocity which was found to be $194 \mathrm{~km} / \mathrm{s}$.

Nonthermal activity of energetic particles was observed at radio wavelengths during the EUV jet evolution. The WAVES instrument [Bougeret et al., 1995] onboard the Wind satellite observed a nonthermal type III radio burst in the radio

\footnotetext{
*DAMTP, Centre for Mathematical Sciences, University of Cambridge, Wilberforce Road, Cambridge, CB3 OWA, UK

${ }^{\dagger}$ Inter-University Centre for Astronomy and Astrophysics, Ganeshkhind, Pune 411007, India.
} 


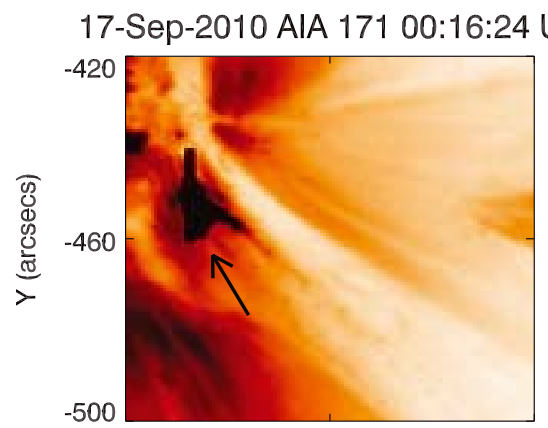

HMI LOS 23:29:06 UT

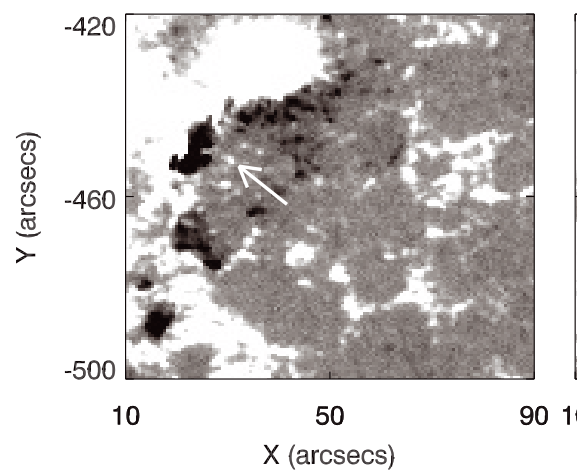

00:21:00 UT

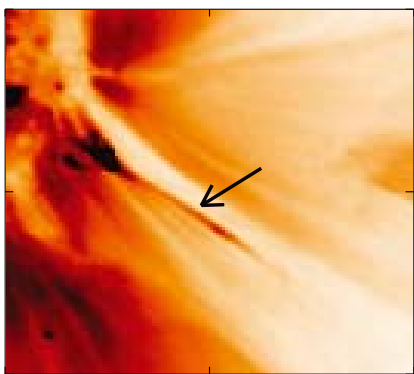

00:21:36 UT

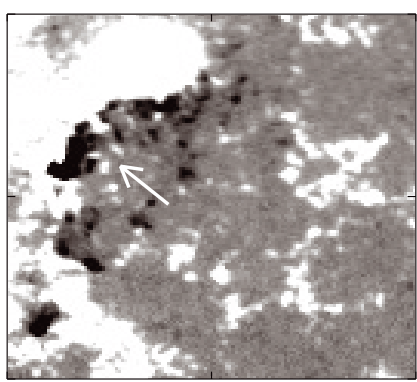

50

$\mathrm{X}(\operatorname{arcsecs})$
00:24:00 UT

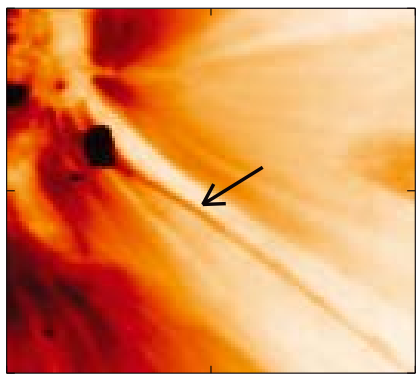

00:59:06 UT

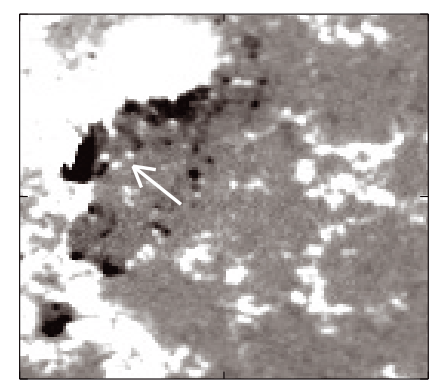

9010

90

$\mathrm{X}(\operatorname{arcsecs})$

Figure 1: Top panel: AIA $171 \AA$ A images of a jet (reverse color) observed on September 17, 2010. The black arrows show the thin spire of the jet. Bottom panel: The photospheric magnetograms observed by the HMI before, during, and after the jet activity. The white arrows show changes in the magnetic activity at the footpoint region of the jet.
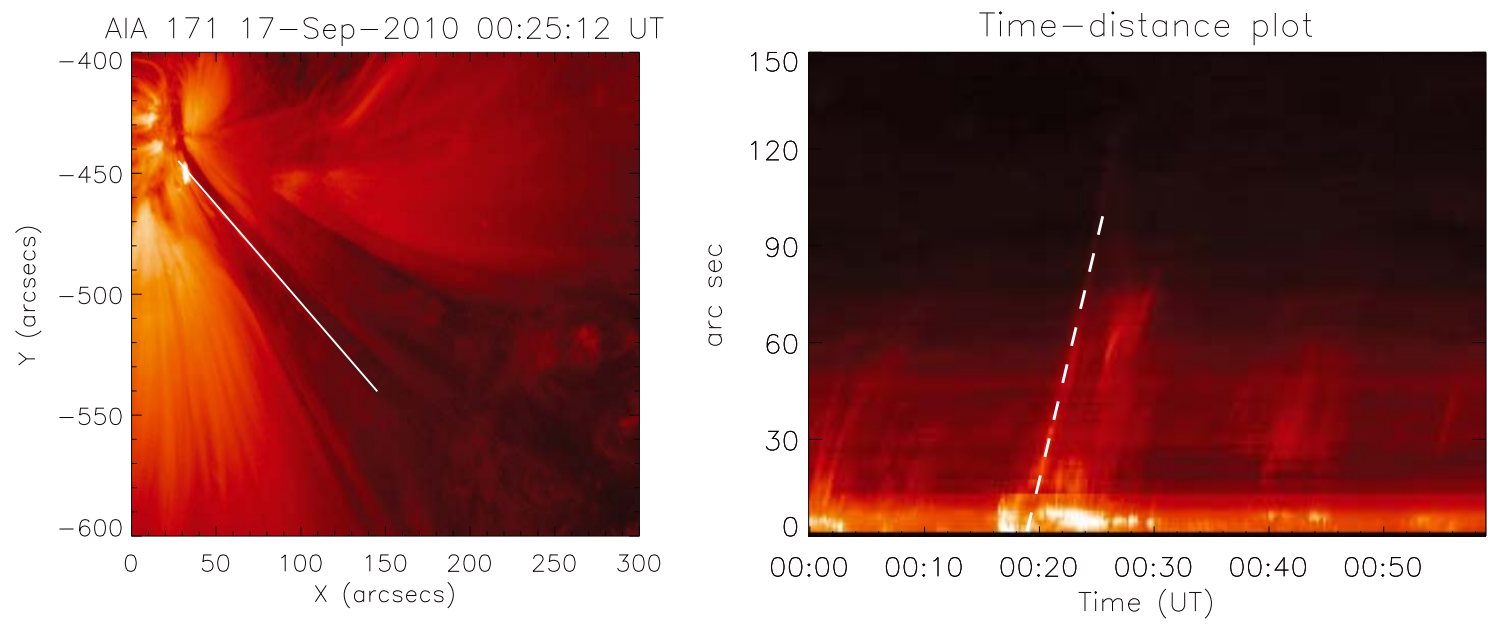

Figure 2: Left panel: The AIA $171 \AA$ image at 00:25 UT, and the white overplotted line indicates the slit position. Right panel: time-distance plot obtained at the slit position. The velocity was calculated at the white dashed line, and it is found to be $194 \mathrm{~km} / \mathrm{s}$. 
dynamic spectrum. Type III bursts are fast drift bursts. They are thought to be produced by the fast gyrating nonthermal electrons along the open magnetic field lines. Figure 3 shows the radio dynamic spectrum of the nonthermal type III radio burst in the frequency range from $13 \mathrm{MHz}$ to $\sim 50 \mathrm{kHz}$. The type III burst (shown by a white arrow) was observed to start at 00:16 UT . The observations showed a temporal correlation between jet activity and nonthermal type III burst.

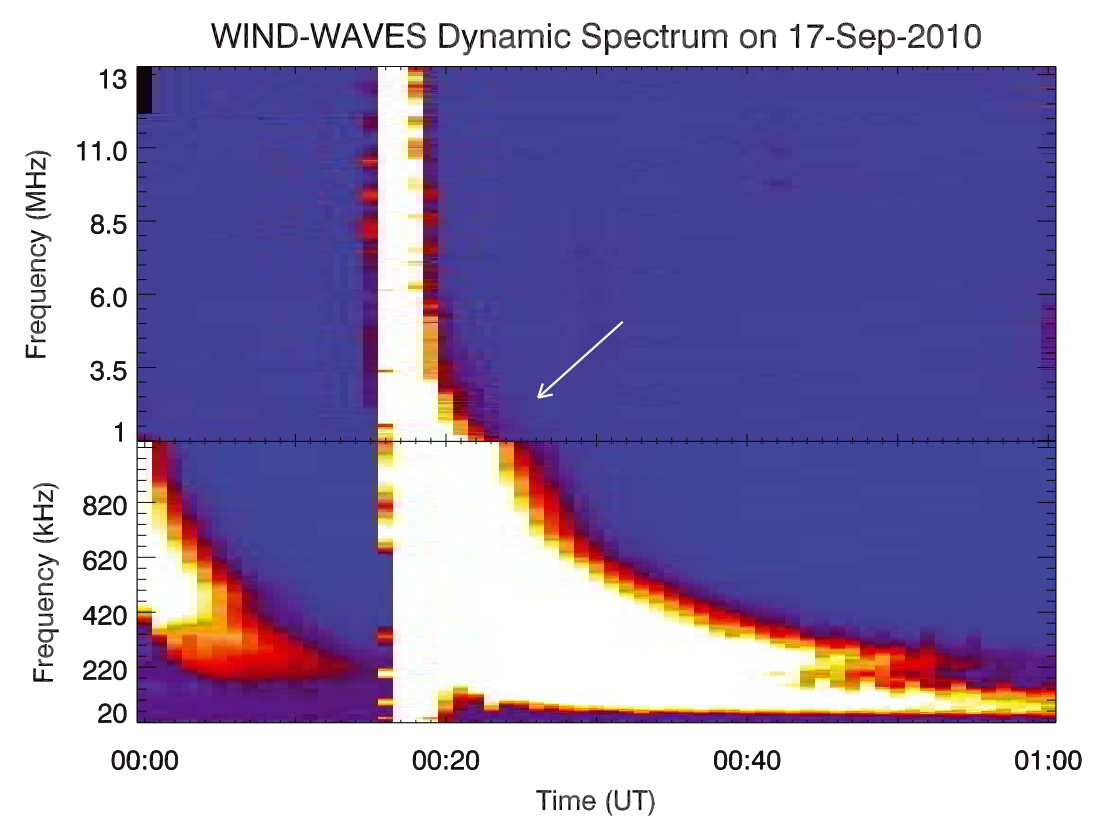

Figure 3: Nonthermal type III radio burst (shown by white arrow) observed by the WAVES instrument.

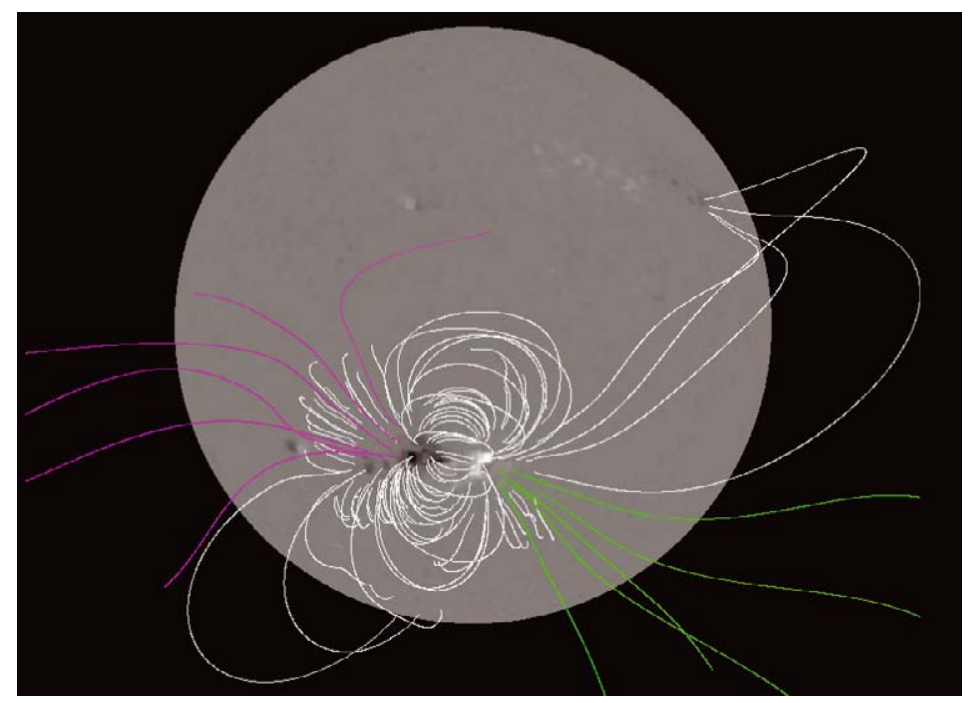

Figure 4: The coronal magnetic field obtained from the PFSS analysis at 00:04 UT. The magnetic field lines associated with an active region are shown by white (closed), green (open positive polarity) and pink (open negative polarity) color. 
Further, we investigated the spatial correlation between the jet and the type III burst by performing a Potential Field Source Surface (PFSS) analysis [Schatten et al., 1969]. The magnetic field in the corona was obtained by extrapolating the photospheric magnetic field. It is believed that type III bursts originate due to propagation of electrons along magnetic field lines. Therefore, the purpose of this is to check whether open magnetic field lines were present at the footpoint region of the jet. Figure 4 shows the PFSS analysis at 00:04 UT, and the coronal magnetic field lines are shown by white (closed), green (open positive polarity) and pink (open negative polarity) lines. The positive polarity (green lines) open field lines were observed on the western edge of the AR at the region of the footpoint of the jet. These observations confirm the spatial correlation between the EUV jet and the nonthermal type III radio burst.

The signatures of nonthermal type III radio bursts associated with active region jets are seen in the interplanetary medium. The observations show an evidence of particle acceleration and an emergence of the photospheric magnetic field before and during the EUV jet activity. Therefore, they are one of the important explosive events to study the Sun-Earth connection.

Acknowledgments. SMM acknowledges financial support from URSI (Union of Radio Science) and the Province of Styria. Also, she acknowledges support from DST under the Fast Track Scheme (SERB/F/3369/2012/2013) and the Cambridge Trust, University of Cambridge, UK. HEM and GDZ acknowledge the support of STFC. AIA data are courtesy of SDO (NASA) and the AIA consortium. The authors thank the open data policy of the WIND/ WAVES instrument team.

\section{References}

Bougeret, J. L., M. L. Kaiser, P. J. Kellogg, R. Manning, K. Goetz, S. J. Monson, N. Monge, L. Friel, C. A. Meetre, C. Perche, L. Sitruk, and S. Hoang, Waves: The Radio and Plasma Wave Investigation on the Wind Spacecraft, Space Sci. Rev., 71, 231-263, 1995.

Lemen, J. R., et al. (46 co-authors), The Atmospheric Imaging Assembly (AIA) on the Solar Dynamics Observatory (SDO), Solar Phys., 275, 17-40, 2012.

Mulay, S. M., D. Tripathi, G. Del Zanna, and H. Mason, Multiwavelength study of 20 jets that emanate from the periphery of active regions, Astron. Astrophys., 589, id.A79, 16 pp., 2016.

Schatten, K.H., J. M. Wilcox, and N.F. Ness, A model of interplanetary and coronal magnetic fields, Solar Phys., 6, 442-455, 1969.

Scherrer, P.H., et al. (12 co-authors), The Helioseismic and Magnetic Imager (HMI) Investigation for the Solar Dynamics Observatory (SDO), Solar Phys., 275, 207$227,2012$. 